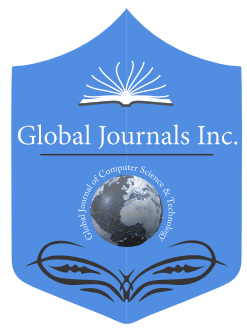

Global JOURnAl of COMPUTER SCIENCE AND TECHNOlOGY: G INTERDISCIPLINARY

Volume 21 Issue 1 Version 1.0 Year 2021

Type: Double Blind Peer Reviewed International Research Journal

Publisher: Global Journals

Online ISSN: 0975-4172 \& Print ISSN: 0975-4350

\title{
A Framework to Model Site Reliability Engineering Implementations and its Consolidation
}

By Anand Sunder

Abstract- Site Reliability Engineering has opened a pandora's box of new technological challenges $[1],[3],[6],[10]$. Trying to condense all of it into one structure or system is one such requirement.

We show the interaction between people, processes, and technology as against the levels of maturity on reliability scale of 5 .

GJCST-G Classification: D.3.3

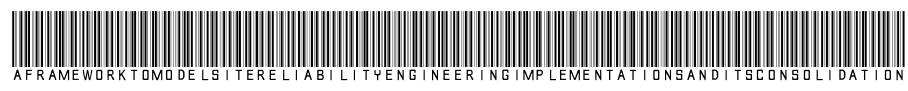

Strictly as per the compliance and regulations of:

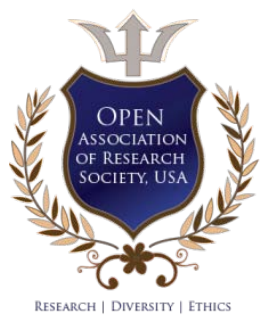

(C) 2021. Anand Sunder. This is a research/review paper, distributed under the terms of the Creative Commons AttributionNoncommercial 3.0 Unported License http://creativecommons.org/licenses/by-nc/3.0/), permitting all non-commercial use, distribution, and reproduction in any medium, provided the original work is properly cited. 


\section{A Framework to Model Site Reliability Engineering Implementations and its Consolidation}

\section{Anand Sunder}

Abstract- Site Reliability Engineering has opened a pandora's box of new technological challenges [1],[3],[6],[10]. Trying to condense all of it into one structure or system is one such requirement.

We show the interaction between people, processes, and technology as against the levels of maturity on reliability scale of $5^{[1]}$.

\section{INTRODUCTION}

W e classify the degree of reliability in systems into five discrete levels as shown below in the table:

Table 1: Discrete levels of classification of SRE maturity levels ${ }^{[1,3]}$

\begin{tabular}{|c|c|}
\hline Level & Type \\
\hline I & Chaotic \\
\hline II & Repeatable \\
\hline III & Defined \\
\hline IV & Managed \\
\hline V & Optimizing \\
\hline
\end{tabular}

Also in lieu of various tenets of Site Reliability Engineering we include the following:

Table 2: Tenets of SRE ${ }^{[1,3,6]}$

\begin{tabular}{|c|c|}
\hline 1 & Monitoring Distributed Systems \\
\hline 2 & Incident Management \\
\hline 3 & Exception Handling \\
\hline 4 & Emergency Response \\
\hline 5 & Toil Elimination \\
\hline 6 & Chaos Engineering \\
\hline
\end{tabular}

Author: e-mail: anand.sunder@capgemini.com
Based on the interaction between people, processes, and technology and the five discrete levels of maturity we come here with a map of their interactions:

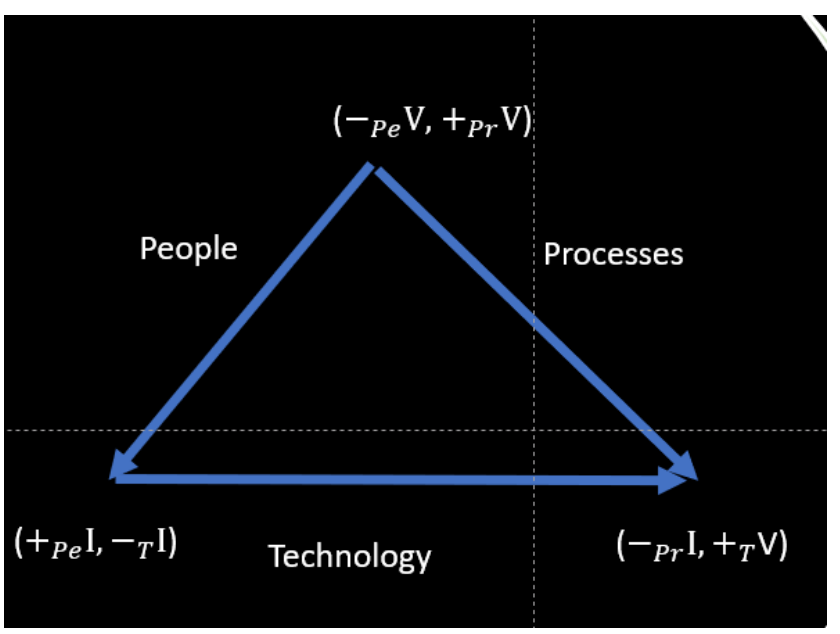

Figure 1: Interaction of people. processes and technology ${ }^{[1,2,3,6,9]}$

Technology bridges the gap between people and processes, so across the discrete levels of maturity, i.e, Levels I $-V$ the above diagram approximates the interactions in most of the cases occurring in real life scenarios.

We have also come up with an approximate flow map of the tenets across 5 discrete levels as shown below:

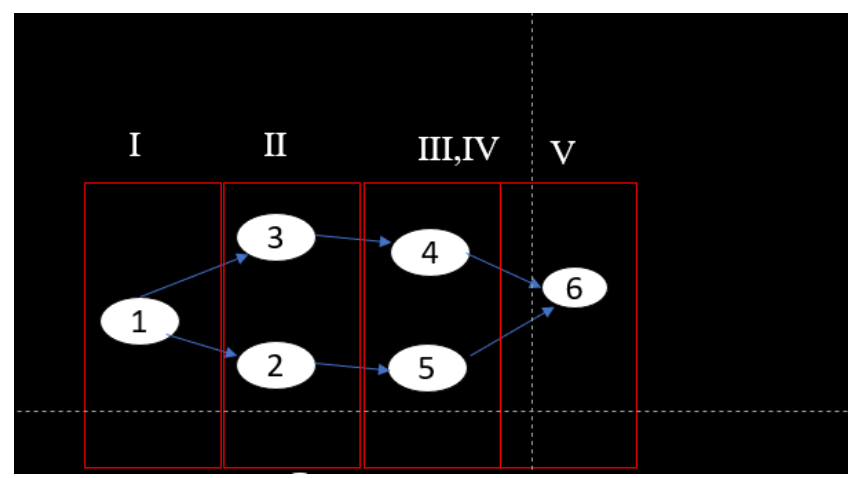

Figure 2: Levels against Tenets of SRE ${ }^{[1,3,6]}$

Monitoring distributed systems reinforces incident management (2) and exception handling (3). 
A mature incident management (2) leads to better elimination of unnecessary toil (5).

An evolved exception handling (3) will lead to an evolved emergency response (4).

Ultimately Levels I-V of maturity will be mapped to various tenets as shown above, with highest level of maturity at chaos engineering (6).

\section{iI. Conclusion}

The abovementioned framework, can lead to some really insightful systemic understanding for improving reliability of services. Further insights and improved level of understanding can be extracted by applying this across multiple scenarios.

\section{ReFerences Références ReFEREnCias}

1. https://cloud.google.com/blog/products/devops-sre /how-to-start-and-assess-your-sre-journey

2. https://n4stack.io/2018/10/04/sre-managed-service/

3. https://en.wikipedia.org/wiki/Reliability_engineering \#: : text=Reliability\%20engineering\%20is\%20a\%20 sub,equipment\%20to\%20function\%20without\%20fail ure.\&text=Reliability\%20is\%20closely\%20related\%2 Oto,moment\%20or\%20interval\%20of\%20time.

4. https://aws.amazon.com/sagemaker/features/

5. https://www.linkedin.com/pulse/top-5-principlessuccessful-sre-transforming-ragu-navaratnam

6. https://www.xenonstack.com/insights/site-reliabilityengineering/

7. https://www.photon.in/what-we-do/sre-bestpractices-guide

8. https://cloud.kapostcontent.net/pub/1418185e-b32 5-49d3-b65c-de338e45cb6f/ebook-10-essentialskills-of-a-site-reliability-engineer-sre.pdf

9. https://www.itnews.com.au/news/anz-to-heavily-em brace-sre-for-new-banking-platform-547128

10. https://www.blueoptima.com/blog/are-site-reliabilityengineers-needed

11. https://www.observability.splunk.com/en_us/infrastr ucture-monitoring/guide-to-sre-and-the-four-goldensignals-of-monitoring.html 